\title{
Multistep Methods Using Higher Derivatives and Damping at Infinity*
}

\author{
By Rolf Jeltsch
}

\begin{abstract}
Linear multistep methods using higher derivatives are discussed. The order of damping at infinity which measures the stability behavior of a $k$-step method for large $h$ is introduced. $A$-stable methods with positive damping order are most suitable for stiff problems. A method for computing the damping order is given. Necessary and sufficient conditions for $A$-stability, $A(\alpha)$-stability and stiff stability are presented. A new $A$-stable two-step method of order 4 with damping order 1 is found and numerical results are given.
\end{abstract}

1. Introduction. Ordinary linear multistep methods have the drawback that there exist no $A$-stable methods with an order larger than 2 (see Dahlquist [11]). By introducing higher derivatives, one can break this order barrier, even if one only uses one-step methods (see [12], [13], [14], [16], [19], [20], [25], [28], [29], [30], [37]). In contrast to earlier work (see [12], [16], [23], [24] , [25], [26], [35] and [36]), this article is devoted to stability properties for large $h$. The order of damping at infinity which measures the stability behavior as $\mu h \longrightarrow-\infty$ is introduced. Here $\mu$ is an eigenvalue of the Jacobian. This damping order is denoted by $\epsilon$. A method with damping order $\epsilon=1$ has exactly the property of damped at $\infty$ introduced by Osborne [33]. An $A$-stable method with positive damping order is stiffly stable defined by Axelsson [2], strongly stable defined by Chipman [7], $L$ stable defined by Lambert [25]. Enright [16] introduced a class of multistep methods using the second derivative with positive damping order. In Section 3 , we give a simple way of computing the order of damping using the so-called Puiseux diagram of a method. Using this result, it is easy to determine which methods are strong candidates for $L$-stability. The coefficients of some of these methods are listed in the microfiche section of this issue. An $A$-stable two-step method with damping order $\epsilon=1$ and error order 4 which uses only $y^{\prime}(x)$ and $y^{\prime \prime}(x)$ is given in Section 4 . Note that a one-step method with the same error order and stability properties can only be found when $y^{\prime \prime \prime}(x)$ is also introduced in the formula. In Section 4 some

Received May 29, 1973; revised July 3, 1974 and December 18, 1975.

AMS (MOS) subject classifications (1970). Primary $65 \mathrm{~L} 05$.

Key words and phrases. Ordinary differential equations, linear $k$-step methods using higher derivatives, Obrechkoff methods, Hermite methods, $A$-stable, order of damping at infinity, stiffly stable, strongly $A$-stable, $L$-stable.

* This work was started when the author was at the Mathematisches Seminar of the Swiss Federal Institute of Technology at Zürich, Switzerland, and was finished during his stay at Dalhousie University. 
necessary conditions for $A$-stability are proved; similar conditions are necessary for $A(\alpha)$-stability introduced by Widlund [39] and stiff stability defined by Gear [18] . Moreover, we give a sufficient condition for $A$-stability. Some numerical examples are presented in Section 5.

2. Preliminaries. Consider the multistep method

$$
\sum_{i=0}^{k} \alpha_{i} \mathrm{y}_{n+i}=\sum_{i=0}^{k} \sum_{j=1}^{l_{i}} h^{j} \beta_{i j} \mathrm{f}_{n+i}^{(j-1)}, \quad n=0,1,2, \ldots,
$$

for solving the initial value problem

$$
\mathbf{y}^{\prime}=\mathbf{f}(x, \mathrm{y}), \quad \mathrm{y}(a)=\eta, \quad x \in I=[a, b),
$$

where $-\infty<a<b \leqslant+\infty, \mathbf{y}, \mathbf{f}(x, \mathbf{y}) \in \mathbf{R}^{m}$. In (2-1) $\mathbf{y}_{n}$ is an approximation to $\mathrm{y}\left(x_{n}\right), x_{n}=a+n h, h>0$ and $\mathbf{f}_{m}^{(j)}=\mathbf{f}^{(j)}\left(x_{m}, \mathbf{y}_{m}\right)$, where

$\mathbf{f}^{(0)}(x, \mathbf{y})=\mathbf{f}(x, y), \quad \mathbf{f}^{(j+1)}(x, \mathbf{y})=\frac{\partial \mathbf{f}^{(j)}(x, \mathbf{y})}{\partial x}+\frac{\partial \mathbf{f}^{(j)}(x, \mathbf{y})}{\partial \mathbf{y}} \mathbf{f}(x, \mathbf{y})$,

$$
j=0,1,2, \ldots
$$

In (2-1) $k$ and $l_{i}, i=0(1) k$, are fixed integers and $\alpha_{i}, \beta_{i j}$ denote real constants. We shall always assume that $\alpha_{k} \neq 0$. We say that (2-1) defines a $(k, l)$-method, where $l:=\max _{i=0(1) k}\left\{l_{i}\right\}$. Moreover, we shall always assume that the functions $\mathrm{f}^{(j)}(x, \mathrm{y})$, $j=0(1)(l-1)$ satisfy a Lipschitz condition in the second variable. If $\Sigma_{j=1}^{l}\left|\beta_{k j}\right|=0$, then the method is called explicit, otherwise implicit.

It is convenient to associate with a $(k, l)$-method the following polynomials

$$
\rho(\zeta):=\sum_{i=0}^{k} \alpha_{i} \zeta^{i} \quad \text { and } \quad \sigma_{j}(\zeta):=\sum_{i=0}^{k} \beta_{i j} \xi^{i}, \quad j=1(1) l,
$$

where we have used the definition

$$
\beta_{i j}:=0, \quad j=l_{i}+1(1) l .
$$

Moreover, we associate with a $(k, l)$-method the operator

$$
L[y, h]:=\left(\rho(E)-\sum_{j=1}^{l} \sigma_{j}(E) h^{j} D^{j}\right) y(x),
$$

where $D$ is the differential operator $D y(x)=d y(x) / d x$ and $E$ is the shift operator $E y(x)=y(x+h)$.

Definition 1. The difference operator (2-3) has (error) order $p$ if for all $y \in$ $C_{I}^{p+1}$

$$
L[y, h]=C_{p+1} h^{p+1} y^{(p+1)}(x)+O\left(h^{p+2}\right),
$$

where $C_{p+1} \neq 0$.

According to Dahlquist $[9$, p. 40], the following assumption can always be made without loss of generality:

(A) The polynomials $\rho(\zeta), \sigma_{j}(\zeta), j=1(1) l$ have no common factor (i.e. there is no complex number $z$ such that $\left.\rho(z)=\sigma_{j}(z)=0, j=1(1) l\right)$. 
The following theorem has been given by Griepentrog [20] (using a different terminology).

THEOREM 1. (a) Each of the following conditions are necessary for the convergence of a $(k, l)$-method.

(i) The zeros of the polynomial $\rho(\zeta)$ lie on the unit disk and the zeros with modulus 1 are simple (condition of stability).

(ii) The associated difference operator (2-3) has at least order 1 (condition of consistency).

(b) $A(k, l)$-method which satisfies (i) and (ii) is convergent.

The two following theorems ensure that there exist convergent methods.

THEOREM 2. To any given real numbers $\alpha_{i}, i=0(1) k$, with $\Sigma_{i=0}^{k} \alpha_{i}=0$ and any integers $l_{i}, i=0(1) k$, there exists a difference operator (2-3) with the given $\alpha_{i}$ with an error order $p \geqslant \sum_{i=0}^{k} l_{i}$.

Theorem 2 can be improved if $k$ is even.

THEOREM 3. Let $k$ be even and let real numbers $\alpha_{i}, i=0(1) k$, with $\alpha_{i}=$ $-\alpha_{k-i}$ and integers $l_{i}, i=0(1) k$ with $l_{i}=l_{k-i}$ be given. If $l_{k / 2}$ is odd, then there exists a difference operator (2-3) with the given $\alpha_{i}$ with an order $p \geqslant q+1$, where $q:=\Sigma_{i=0}^{k} l_{i}$.

Reimer [35] has shown that the error order $p$ of a convergent $(k, l)$-method satisfies

$$
\begin{array}{ll}
p \leqslant l(k+1)+1 & \text { if } k \text { even, } l \text { odd, } \\
p \leqslant l(k+1) & \text { otherwise. }
\end{array}
$$

Coefficients of methods with $l_{i}=l, i=0(1) k$ have been computed by Lambert and Mitchell [26] for all cases with $k+l \leqslant 5$. The following $(k, 2)$-methods have been introduced by Enright [16] .

Example 1. Enright's methods. In these methods $l=l_{k}=2, l_{i}=1, i=$ $0(1)(k-1)$ and $\alpha_{k}=1, \alpha_{k-1}=-1, \alpha_{i}=0, i=0(1) k-2$. These methods are given by the formula

$$
\begin{aligned}
y(x+k h)-y(x+(k-1) h)= & h \sum_{i=1}^{k}\left(\gamma_{i}^{*}-\frac{k+1}{i} \gamma_{k+1}^{*}\right) \nabla^{i} y^{\prime}(x+k h) \\
& +h \gamma_{0}^{*} y^{\prime}(x+k h)+h^{2}(k+1) \gamma_{k+1}^{*} y^{\prime \prime}(x+k h) \\
& +h^{k+3}\left(\gamma_{k+2}^{*}-\frac{k+1}{k+2} \gamma_{k+1}^{*}\right) y^{(k+3)}(x+k h) \\
& +O\left(h^{k+4}\right),
\end{aligned}
$$

where $\nabla^{i}$ is the $i$ th power of the backward difference $\nabla y(x+k h)=y(x+k h)-$ $y(x+(k-1) h)$. The coefficients $\gamma_{j}^{*}$ in $(2-6)$ are defined by

$$
\gamma_{j}^{*}:=(-1)^{j} \int_{-1}^{0}\left(\begin{array}{c}
-s \\
j
\end{array}\right) d s, \quad j=0(1) \infty
$$

(see Henrici [21, p. 194]). 
3. The Order of Damping at Infinity. The condition of stability introduced in Section 2 is necessary for the convergence of $(k, l)$-methods. Hence, it describes the stability as $h$ tends to zero. The following sections are concerned with methods for stiff differential equations, i.e. systems of differential equations where the eigenvalues of the Jacobian lie in the left-hand plane and have real parts which differ greatly in magnitude. In connection with stiff differential equations one usually considers the scalar test initial value problem

$$
y^{\prime}=\mu y, \quad y(0)=1, \quad x \in I=[0, \infty),
$$

where $\mu$ is an arbitrary complex number. When we apply a $(k, l)$-method to the problem (3-1) then we have the recurrence relation

$$
\sum_{i=0}^{k} \alpha_{i} y_{n+i}-\sum_{i=0}^{k} \sum_{j=1}^{l_{i}}(h \mu)^{j} \beta_{i j} y_{n+i}=0, \quad n=0(1) \infty .
$$

This is a linear homogeneous difference equation with constant coefficients and its characteristic equation is

$$
\phi(\zeta, \lambda)=\rho(\zeta)-\sum_{j=1}^{l} \lambda^{j} \sigma_{j}(\zeta)=0, \quad \lambda:=h \mu .
$$

This is an algebraic equation and hence there exist $k$ solutions $\zeta_{i}(\lambda), i=1(1) k$. The function $\zeta_{i}(\lambda)$ are branches of an algebraic function. Every solution of (3-2) can be written in the form

$$
y_{n}=\sum_{i=1}^{k} \pi_{i}(n) \zeta_{i}^{n}(\lambda)
$$

where $\pi_{i}(x)$ is a polynomial of degree less than the multiplicity of the zero $\zeta_{i}(\lambda)$. The * in the sum of (3-4) indicates that the sum is only taken over those $i$ which have a finite $\zeta_{i}(\lambda)$. Using (3-4), it is evident that the following lemma holds.

Lemma 1. $A(k, l)$-method is $A$-stable if and only if $\max _{j=1(1) k}\left\{\left|\zeta_{j}(\lambda)\right|\right\}<1$ whenever $\operatorname{Re} \lambda<0$.

To measure the behavior of the numerical solution when $\lambda$ is in a neighborhood of $-\infty$ we introduce the following

Definition 2. A $(k, l)$-method is damped at infinity of order $\epsilon$ if

$$
\max _{i=1(1) k}\left\{\left|\zeta_{i}(\lambda)\right|\right\}=O\left(\frac{1}{\lambda^{\epsilon}}\right)
$$

as $\lambda$ tends to infinity, that is $|\lambda|^{\eta} \max _{i=1(1) k}\left\{\left|\zeta_{i}(\lambda)\right|\right\}$ is bounded in a neighborhood of $\infty$ for all $\eta \leqslant \epsilon$.

Osborne [33, p. 201] calls a method with $\epsilon=1$ "damped at $\infty$." An $A$-stable method with a positive $\epsilon$ is called stiffly $A$-stable, Axelsson [2, p. 186], strongly stable, Chipman [7, p. 13]. Lambert [25, p. 236] following Ehle [14, p. 7] calls these methods $L$-stable. An $A$-stable method has $\epsilon \geqslant 0$, but $\epsilon \geqslant 0$ does not imply $A$-stability as can be seen from the following example:

$$
y_{n+3}=\frac{1}{11}\left\{18 y_{n+2}-9 y_{n+1}+2 y_{n}-h 6 f_{n+3}\right\} \text {. }
$$


This method has $\epsilon=1 / 3$ as we will prove in Theorem 5. Since the error order of (3-6) is 3, it cannot be $A$-stable (see Dahlquist [11, p. 31]).

Definition 3. Let a $(k, l)$-method fulfill (A). The set

$$
P:=\left\{(i, 0) \mid \text { if } \alpha_{i} \neq 0\right\} \cup\left\{(i, j) \mid \text { if } \beta_{i j} \neq 0\right\}
$$

is called the Puiseux set of the method. The graph of $P$ in $R^{2}$ is called the associated Puiseux diagram and is denoted by $G(P)$.

Example 2. The Puiseux diagram $G(P)$ of Enright's $(3,2)$-method, described in Example 1, consists of all points in Figure 1 represented by an asterisk.

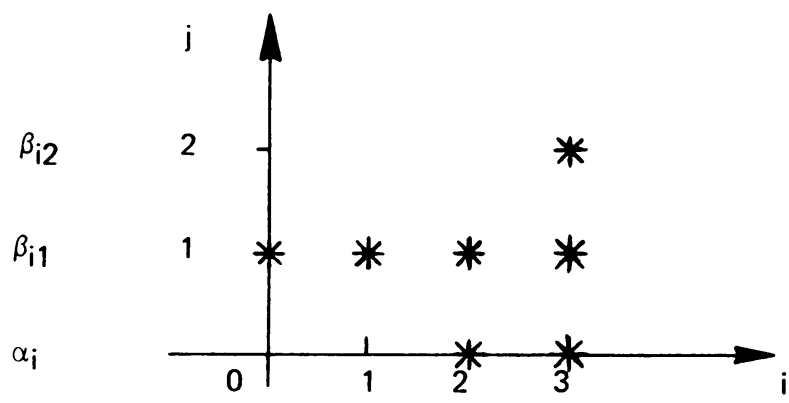

FIgURE 1. Puiseux diagram of Enright's (3, 2)-method

Without loss of generality we can always assume that

$$
\text { if } l_{i}>0 \quad \text { then } \beta_{i l_{i}} \neq 0, i=0(1) k \text {. }
$$

The following theorem provides an easy way to calculate the damping order $\epsilon$.

THEOREM 4. Let a $(k, l)$-method fulfill (A) and (B) and let $G(P)$ be its

Puiseux diagram. Then the damping order $\epsilon$ is the smallest slope of all straight lines having a finite slope and passing through $\left(k, l_{k}\right)$ and any other point of $G(P)$.

Proof. We write (3-3) in the form

$$
\phi(\zeta, \lambda)=\sum_{i=0}^{k} \eta_{i}(\lambda) \zeta^{i}=0
$$

where

$$
\eta_{i}(\lambda):=\sum_{j=0}^{l_{i}} \eta_{i j} \lambda^{j}:=\alpha_{i}-\sum_{j=1}^{l_{i}} \beta_{i j} \lambda^{j}, \quad i=0(1) k .
$$

It is well known, that (3-7) has $k$ solutions $\zeta_{i}(\lambda), i=1(1) k$, which can be represented in a neighborhood of $\lambda=\infty$ by the convergent series

$$
\zeta_{i}(\lambda)=e_{1 i} \lambda^{-\epsilon} 1 i+e_{2 i} \lambda^{-\epsilon} 2 i+e_{3 i} \lambda^{-\epsilon} 3 i+\cdots,
$$

see, e.g. Ahlfors [1]. Here, $-\infty<\epsilon_{1 i}<\epsilon_{2 i}<\epsilon_{3 i}<\cdots$ and $e_{1 i} \neq 0, i=1(1) k$.

Hence, we have the damping order

$$
\epsilon=\min _{i=1(1) k}\left\{\epsilon_{1 i}\right\}
$$

In the following we compute the smallest $\epsilon_{1 i}$. In order to simplify the notation we 
omit the second index. Since (3-7), we have

$$
\begin{aligned}
\eta_{k}(\lambda)\left(e_{1} \lambda^{-\epsilon}+e_{2} \lambda^{-\epsilon} 2\right. & +\cdots)^{k}+\eta_{k-1}(\lambda)\left(e_{1} \lambda^{-\epsilon_{1}}+e_{2} \lambda^{-\epsilon_{2}}+\cdots\right)^{k-1} \\
& +\cdots+\eta_{1}(\lambda)\left(e_{1} \lambda^{-\epsilon_{1}}+e_{2} \lambda^{-\epsilon_{2}}+\cdots\right)+\eta_{0}(\lambda) \equiv 0 .
\end{aligned}
$$

The series

$$
\zeta(\lambda)=e_{1} \lambda^{-\epsilon_{1}}+e_{2} \lambda^{-\epsilon_{2}}+e_{3} \lambda^{-\epsilon_{3}}+\cdots
$$

is absolute convergent in a neighborhood of $\lambda=\infty$, and hence we can expand each $\eta_{i}(\lambda) \zeta^{i}(\lambda)$ in (3-11) in a series in powers of $\lambda$. Since the sequence $\epsilon_{n}, n=1(1)^{\infty}$ is strictly increasing, there are terms on the left-hand side of (3-11) with a power of $\lambda$ which is higher than in all other terms; and the sum over these terms has to vanish. Hence, among the terms

$$
\eta_{k l_{k}} e_{1}^{k} \lambda^{l_{k}-k \epsilon_{1}}, \eta_{k-1 l_{k-1}} e_{1}^{k-1} \lambda^{l_{k-1}-(k-1) \epsilon_{1}}, \ldots, \eta_{1 l_{1}} e_{1} \lambda^{l_{1}-\epsilon_{1}}, \eta_{0 l_{0}} \lambda^{l_{0}}
$$

there are at least two with the same power of $\lambda$ which is larger than all other powers. This gives us a condition for $\epsilon_{1}$, and we have therefore by $(3-10)$ to look for the smallest $\epsilon_{1}$ such that at least two of the numbers

$$
\left\{l_{i}-i \epsilon_{1} \mid \text { if } l_{i}>0 \text { or } \alpha_{i} \neq 0, i=0(1) k\right\}
$$

are equal and larger than all others. We draw through each point of the set

$$
\{(i, j) \in P \mid(i, j+t) \notin P, t=1(1) \infty\}
$$

a straight line with the slope $\epsilon_{1}$; see Figure 2.

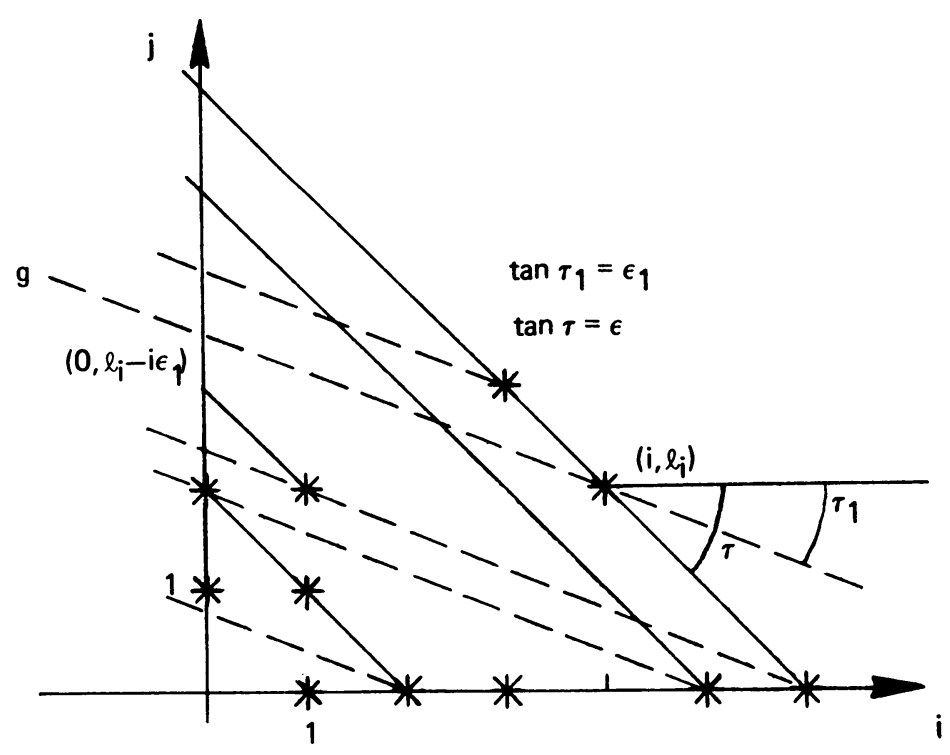

Figure 2

Let, for instance, $g$ be the line passing through $\left(i, l_{i}\right)$; then $g$ passes through the vertical axis at the point $\left(0, l_{i}-i \epsilon_{1}\right)$. Therefore, we have to look for the smallest 
slope $\epsilon_{1}$ such that at least two of these lines are identical; and all others have a smaller ordinate at $x=0$. Clearly, this slope will be $\epsilon$ and can be found as described in Theorem 4.

Clearly the following corollary holds.

Corollary 1. Let a $(k, l)$-method fulfill (A) and (B). Then

$$
\epsilon=\min \left\{\frac{l_{k}-l_{i}}{k-i} \mid \text { if } l_{i}>0 \text { or } \alpha_{i} \neq 0, i=0(1) k-1\right\} \text {. }
$$

Example 3. We consider Enright's methods described in Example 1. By (2-7) one finds $\gamma_{0}^{*}=1, \gamma_{j}^{*}<0$ and $j \gamma_{j}^{*}-(j+1) \gamma_{j+1}^{*}<0, j=1(1) \infty$. Hence, $\beta_{01} \neq 0$ and $\beta_{k 2} \neq 0$. The Puiseux diagram has, therefore, the form given in Figure 3.

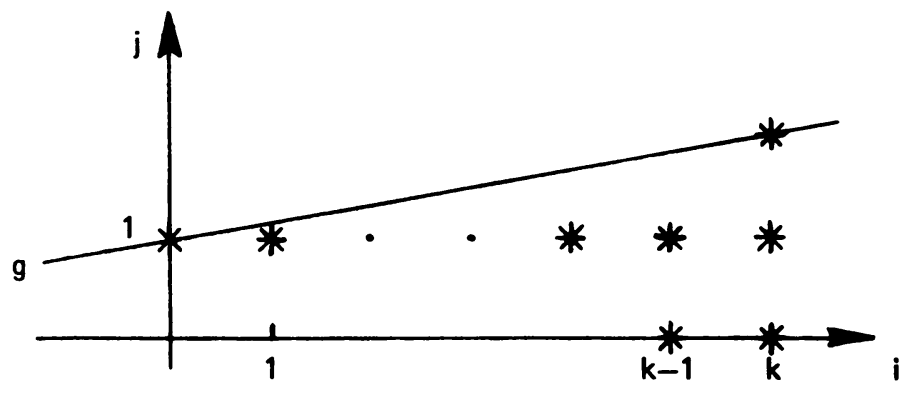

Figure 3. Puiseux diagram of Enright's $(k, 2)$-method

By Theorem 4, $\epsilon$ is equal to the slope of the line $g$ in Figure 3, and hence $\epsilon=1 / k$.

Using techniques similar to those of Example 3, it can be easily proved that $\epsilon$ has the values given in Table 1. The methods are described in Henrici [21] as well as the different parameters.

Example 4. (1, $l)$-methods. For a convergent $(1, l)$-method with $(\mathrm{A})$ and $(\mathrm{B})$ we have by Corollary 1

$$
\epsilon=l_{1}-l_{0}
$$

This can also be seen directly from (3-7), since

$$
\zeta(\lambda)=-\eta_{0}(\lambda) / \eta_{1}(\lambda)
$$

By (3-15), $\zeta(\lambda)$ is a rational function and from (2-4) it follows that

$$
-\eta_{0}(\lambda) / \eta_{1}(\lambda)=e^{\lambda}+O\left(\lambda^{p+1}\right)
$$

Here $p$ is the order of the $(1, l)$-method. Hence, each rational approximation to $e^{\lambda}$ with $p \geqslant 1$ gives rise to a $(1, l)$-method and vice versa, e.g. let $E_{m n}$ be the $(m, n)$ Padé approximation to $e^{\lambda}$, then the corresponding methods have the following properties, listed in Table 2.

For other $(1, l)$-methods see Davison [13], Liniger, Willoughby [28], Loscalzo [29], Makinson [30], Thompson [37] and for suitable rational approximations to $e^{\lambda}$ see Blue, Gummel [4], Cavendish, Culham, Varga [6], Lawson [27], Varga [38].

The proof of the following theorem will be omitted since it follows easily from Theorem 4. 
TABLE 1. Damping order $\epsilon$ of (., 1)-methods

\section{Name of method}

Adams-Bashforth

Adams-Moulton

Nyström

Milne-Simpson ${ }^{1}\left\{\begin{array}{l}q=0 \\ q=1 \\ q=2 \\ q>2\end{array}\right.$

\begin{tabular}{l|l}
$\begin{array}{l}\text { Method based } \\
\text { on differen- } \\
\text { tiation at the } \\
\text { point } x_{n+k-r}\end{array}$ & $r=1, q>0$ \\
& $r>1, q=1$
\end{tabular}

Optimal methods

(in the sense of

Dahlquist)

(., 1)-method

$\epsilon$

error order

$\begin{array}{rrr}k & -1 & k \\ k & 0 & k \\ k & -1 & k \\ 1 & 1 & 1 \\ 2 & -1 & 2 \\ 2 & 0 & 4 \\ k=q & 0 & k+1\end{array}$

$\begin{array}{lrr}k & \frac{1}{k} & k \\ k=q & -1 & k \\ r & -\frac{1}{k} & 1\end{array}$

$k$

0

$k+2$

\footnotetext{
${ }^{1}$ The Milne-Simpson methods are described by the formula $y_{n}-y_{n-2}=n \Sigma_{m=0}^{q}{ }_{m}^{*} \nabla^{m} f_{n}$ where the constants $\kappa_{m}^{*}$ are given in Henrici $[21$, p. 201$]$.
}

TABLE 2. Damping order $\epsilon$ of $(1, l)$-methods corresponding to the Pade approximation

Method such that

$-\eta_{0}(\lambda) / \eta_{1}(\lambda)=\cdots$

(1, .)-method

$\epsilon$

error order

$A$-stability

$E_{l l}$

$l$
$l$
$l$
$l$

0

$2 l$

yes 1)

$E_{l l-1}$

$1 \quad 2 l-1$

yes 2)

$E_{l l-2}$

2

$2 l-2$

yes 2)

$E_{l-3}$

$3 \quad 2 l-3$

no 2)

$E_{l 0}, l=3,4,5$

$l$

no 3)

1) Birkhoff, Varga [3] , 2) Ehle [14] , 3) Calahan [5].

THEOREM 5. The order of damping at infinity of a $(k, l)$-method with (A), (B) and $\eta_{0}(\lambda) \equiv 0$ satisfies

$$
\epsilon \leqslant l / k
$$


In (3-17) we have equality if and only if

$$
l_{k}=l, \quad l_{0}=0, \quad \alpha_{0} \neq 0
$$

and

$$
l_{i} \leqslant\left[i \frac{l}{k}\right] \quad \text { if } \eta_{i}(\lambda) \neq \equiv 0, i=1(1) k-1 .^{* *}
$$

$\mathrm{A}(k, l)$-method which fulfills (A), (B) and (3-18) is called a $(k, l)$-method with optimal damping order. The $(k, 1)$-methods with optimal $\epsilon$ and highest order are the methods based on differentiation with $r=0$ (see Henrici [21, p. 206]). These methods are only convergent for $k=1(1) 6$ (see Cryer [8]), but for $k \leqslant 6$ they are very efficient for solving stiff differential equations (see Gear [18]). Using techniques given in Jeltsch [24], it can be shown that to any given $k, l$ and $\epsilon$ there exists a unique $(k, l)$-method with damping order $\epsilon$ and an error order which is larger than the error order of any other $(k, l)$-method with damping order $\epsilon$. For $\epsilon>0$ these methods have been determined by solving the linear system of equations which arises from (2-4) (see, e. g. Lambert and Mitchell [26]). The computation was carried out by a SYMBAL-program.*** The coefficients of the methods together with the error order and $C_{p+1}$ are listed in Tables 3 and 4 for $k=1,2,3 ; l=1,2,3,4$ and $k=4, l=$ $1,2,3$. The tables appear in the microfiche section of this issue. All except four of the methods are stable and hence convergent. The results in Table 3, together with the results of Dahlquist [10], and Cryer [8] and Reimer [35], seem to suggest that to each $(k, l)$ there might exist a critical $\epsilon_{k, l}$ such that to a given damping order $\epsilon$ the $(k, l)$-method of highest error order is stable if and only if $\epsilon \geqslant \epsilon_{k, l}$.

4. Necessary and Sufficient Conditions for $A$-Stability. We shall prove the following.

THEOREM 6. Let a $(k, l)$-method fulfill (A) and (B). The following conditions are necessary for A-stability.

(i) $\epsilon \geqslant 0$.

(ii) $l=l_{k}$.

(iii) If $\epsilon=0$, then the zeros of the polynomial $\sigma_{l}(\zeta)$ lie on the closed unit disk.

(iv) The zeros of $\eta_{k}(\lambda)$ have a positive real part.

(v) $\max _{j=1(1) k}\left\{\left|\zeta_{j}(i y)\right|\right\} \leqslant 1$ for all $y \in R$.

Moreover, (iv) and (v) together are sufficient for A-stability.

Proof. (i) is a trivial consequence of Definition 2 and Lemma 1, and (ii) follows from (i) using Corollary 1. To show (iii) we look at the leading term $e_{1} \lambda^{-\epsilon_{1}}$ of the series (3-12). Since $\epsilon_{1}=\epsilon=0$, the term in (3-11) belonging to the highest power of $\lambda$, namely $\lambda^{0}$, can be written as $\sigma_{l}\left(e_{1}\right)$. This term has to vanish and hence $e_{1}$ is a zero of $\sigma_{l}(\zeta)$. By Lemma 1 and (3-9), (iii) follows immediately. To show (iv) let $\lambda_{1}$ be a zero of $\eta_{k}(\lambda)$. If $\lambda=\lambda_{1}$, then (3-7) reduces to $\sum_{i=0}^{k-1} \eta_{i}(\lambda) \zeta^{i}$ which is a polynomial in

${ }^{* *}[a]$ denotes the largest integer not exceeding $a$.

***SYMBAL is a formula manipulation language. For a description see Engeli [15]. The computation was carried out on the CDC $6400 / 6500$ of the Swiss Federal Institute of Technology at Zürich. 
$\zeta$ of degree $k-1$ or less. Hence, one of the $k$-solutions $\zeta_{i}(\lambda), i=1(1) k$ of (3-7) tends to infinity as $\lambda$ tends to $\lambda_{1}$. This establishes (iv). That (v) is necessary is trivial since by Lemma 1 the algebraic functions $\zeta_{j}(\lambda)$ can have no poles on $\lambda=i y, y \in R$ and hence are continuous in a strip containing the imaginary axis. To prove that (iv) and (v) are sufficient for $A$-stability we first observe that by (iv) the functions $\zeta_{j}(\lambda)$ have no poles in the left-hand plane since $\eta_{k}(\lambda) \neq 0$ whenever $\operatorname{Re} \lambda \leqslant 0$. Moreover, since the $\zeta_{j}(\lambda)$ are algebraic, (v) implies that we have no pole at $\lambda=\infty$; in fact, we have

$$
\max _{j=1(1) k}\left\{\left|\zeta_{j}(\infty)\right|\right\} \leqslant 1
$$

The $\zeta_{j}(\lambda)$ can be considered as the $k$ branches of an algebraic function on a Riemann surface. By the maximum principle for analytic functions on Riemann surfaces (see, e.g. Pfluger [34, p. 17]) and the continuity of algebraic functions (v) together with (4-1) implies that either $\left|\zeta_{j}(\lambda)\right|<1, j=1(1) k$ whenever $\operatorname{Re} \lambda<0$ or $\zeta_{j}(\lambda)=$ constant. However, $\zeta_{j}(\lambda)=$ constant implies that $\rho(\zeta)$ and $\sigma_{j}(\zeta), j=1(1) l$ have a common factor, and this is a contradiction to (A). Hence, by Lemma 1 the method is $A$-stable. This completes the proof of Theorem 6 .

The condition (ii) in Theorem 6 contains as a special case the result of Dahlquist [11] that an explicit, $(k, 1)$-method cannot be $A$-stable. Clearly, (i)-(iii) are also necessary conditions for stiff stability defined by Gear [18] and $A(\alpha)$-stability defined by Widlund [39]. In the same way as we proved (iv) in Theorem 6 one can show that the zeros of $\eta_{k}(\lambda)$ cannot lie in the region of absolute stability $\Omega:=$ $\left\{\lambda\left|\max _{i=1(1) k}\right| \zeta_{i}(\lambda) \mid<1\right\}$. The conditions (i)-(iv) are not sufficient for $A$-stability since the (3,1)-method given in (3-6) fulfills (i)-(iv), $\eta_{3}(\lambda)=1-6 \lambda / 11$, and is not $A$-stable. Using Theorem 6 , it is easy to show that Enright's $(2,2)$-method and the following method,

$$
17 y_{n+2}-16 y_{n+1}-y_{n}=h\left(10 f_{n+2}+8 f_{n+1}\right)-2 h^{2} f_{n+2}^{(1)}
$$

are $A$-stable. Condition (iv) is trivially satisfied since $(-1)^{j} \eta_{k j}>0, j=0,1,2$. Condition (v) can be verified by making the transformation $\zeta=(z+1) /(z-1), z=$ $(\zeta+1) /(\zeta-1)$ and using a theorem due to Frank (see, e.g. Marden [31, p. 179]). We omit the details since it consists only of tedious computations. For brevity we refer to these methods as E4 and J4. Both are $L$-stable since the damping order is $\epsilon=1 / 2$, $\epsilon=1$, respectively. The error order of both methods is 4 . The author knows no other $L$-stable $(k, 2)$-method with an order exceeding 3.

5. Numerical Illustration. In the following examples we compare the methods $\mathrm{J} 4, \mathrm{~J} 5$ and E4. J5 is given by the formula

$$
23 y_{n+2}-16 y_{n+1}-7 y_{n}=h\left(12 f_{n+2}+16 f_{n+1}+2 f_{n}\right)-2 h^{2} f_{n+2}^{(1)}
$$

(see also Tables 3 and 4 of the microfiche section of this issue). J5 is not $A$-stable since $\phi(-1, \lambda)=0$ has a root in the left-hand plane. However, it is easily verified that $\mathrm{J} 5$ is $A_{0}$-stable (i.e. $\left|\zeta_{j}(x)\right|<1, j=1,2$ whenever $x \in(-\infty, 0)$ ). J5 has error order 5 and $\epsilon=1 / 2$. 
FIGURE 4. Relative error $E$ at $x=32$ as a function of the stepsize $h$

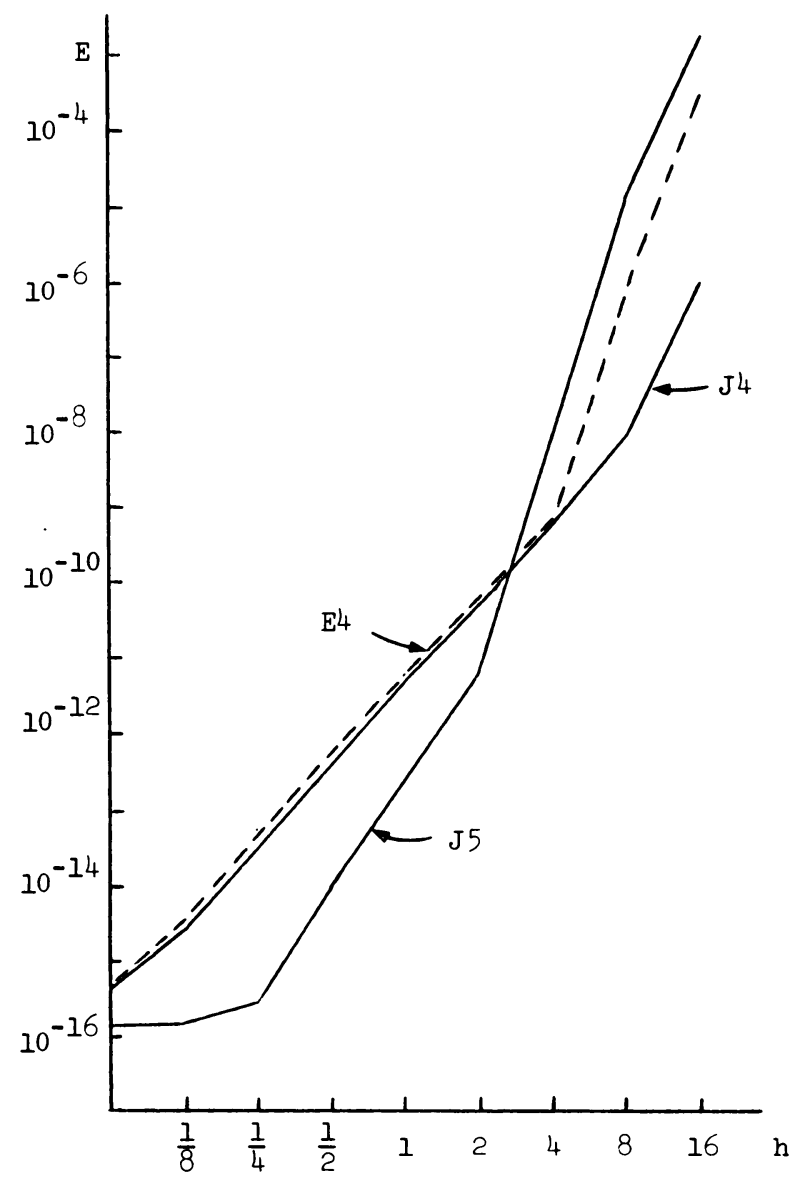

Example 5. Consider the initial value problem proposed by Gear [18]:

$$
y^{\prime}=\lambda(y-g(x))+g^{\prime}(x), \quad y(0)=g(0)+c .
$$

The exact solution is $y(x)=g(x)+c e^{\lambda x}$. Here $c, \lambda$ are arbitrary parameters and $g(x)$ an arbitrary function in $C^{1}[0, \infty)$. If $\lambda$ is much smaller than zero and $g(x)$ is smooth, then the problem is stiff. We choose $c=1, \lambda=-20$ and $g(x)=\arctan (x)$. The exact solution is $y(x)=\arctan (x)+e^{-20 x}$. The methods are started with exact starting values. At each step the implicit equation (2-1) for $y_{n+k}$ is solved analytically. The numerical results are given in Table 5 and Figure 4 where $E_{n}$ denotes the relative error, that is $E_{n}=\left|\left(y_{n}-y\left(x_{n}\right)\right) y\left(x_{n}\right)^{-1}\right|$. One observes in Figure 4 that for $h<2$ the methods behave as predicted by the classical theory (see, e.g. Henrici [21]); that is, the higher order method J5 is better than the lower order methods E4 and J4. Moreover, of the two methods of order 4 the one with the smaller error constant

$$
C=C_{p+1} / \sigma_{1}(1)
$$

(see Henrici [21, p. 223]) is better. One has $C=1 / 270$ for $\mathrm{J} 4$ and $C=7 / 1440$ 
TABLE 5. Relative error $E_{n}{ }^{1}$ of $\mathrm{J} 4, \mathrm{~J} 5$ and $\mathrm{E} 4$ as a function of $x$ for Example 5

\begin{tabular}{|c|c|c|c|c|c|c|c|}
\hline \multirow{2}{*}{\multicolumn{2}{|c|}{$\frac{\text { Method }}{\mathrm{h}}$}} & \multicolumn{2}{|c|}{ E4 } & \multicolumn{2}{|c|}{$\mathrm{J} 4$} & \multicolumn{2}{|l|}{ J5 } \\
\hline & & 1 & 2 & 1 & 2 & 1 & 2 \\
\hline$x_{n}$ & $\mathrm{y}\left(\mathrm{x}_{\mathrm{n}}\right)$ & $E_{n}$ & $E_{n}$ & $E_{n}$ & $E_{n}$ & $E_{n}$ & $E_{n}$ \\
\hline 2 & 1.107 & $6.1(-2)$ & - & $9.5(-3)$ & - & $2.8(-1)$ & - \\
\hline 4 & 1.326 & $1.0(-3)$ & $2.8(-2)$ & $1.6(-4)$ & $1.6(-3)$ & $1.2(-2)$ & $1.5(-1)$ \\
\hline 6 & 1.406 & $2.2(-5)$ & $1.9(-3)$ & $2.1(-6)$ & $1.7(-4)$ & $7.4(-6)$ & $2.4(-2)$ \\
\hline 8 & 1.446 & $2.6(-7)$ & $2.1(-4)$ & $1.6(-7)$ & $9.1(-6)$ & $1.0(-5)$ & $1.2(-3)$ \\
\hline 10 & 1.447 & $6 \cdot 3(-8)$ & $2 \cdot 3(-5)$ & $5.8(-8)$ & $1.9(-6)$ & $2.2(-7)$ & $2.5(-4)$ \\
\hline 32 & 1.540 & $5.6(-11)$ & $6.0(-10)$ & $4.6(-11)$ & $4.9(-10)$ & $2.4(-12)$ & $5.8(-11)$ \\
\hline 64 & 1.555 & $6.8(-13)$ & $8.2(-12)$ & $6.7(-13)$ & $6.8(-12)$ & $1.6(-14)$ & $3.9(-13)$ \\
\hline 124 & 1.5627 & $1.7(-14)$ & $1.5(-13)$ & $1.4(-14)$ & $1 \cdot 3(-13)$ & $1.4(-15)$ & 0 machine \\
\hline 126 & 1.5629 & $1.6(-14)$ & $1.3(-13)$ & $1 \cdot 3(-14)$ & $1.1(-13)$ & $1.4(-15)$ & $1.4(-15)$ \\
\hline 128 & 1.5630 & $1.4(-14)$ & $1: 2(-13)$ & $1.1(-14)$ & $1.1(-13)$ & $1.4(-15)$ & 0 machine \\
\hline
\end{tabular}

1 The integer in parenthesis indicates powers of 10 by which the preceding numbers are to be multiplied.

TABLE 6. Number of Newton iterations used while integrating with $\mathrm{J} 4$ from $x=0$ to $x=48(\mathrm{~N}=$ total number of iterations, $\mathrm{A}=N \mathrm{~h} /(48-h)=$ average number of iterations per integration step)

\begin{tabular}{rrrrrrrrrrrrrr}
\hline \multicolumn{1}{l}{} & $2^{-7}$ & $2^{-6}$ & $2^{-5}$ & $2^{-4}$ & $2^{-3}$ & $2^{-2}$ & $2^{-1}$ & 1 & 2 & 4 & 8 & 16 \\
\hline $\mathrm{N}$ & 12289 & 6145 & 3073 & 1537 & 961 & 573 & 285 & 142 & 70 & 45 & 24 & 13 \\
$\mathrm{~A}$ & 2.0005 & 2.0009 & 2.002 & 2.004 & 2.5 & 3.0 & 3.0 & 3.02 & 3.04 & 4.09 & 4.8 & 6.5 \\
\hline
\end{tabular}


FIGURE 5. $\mathrm{E}=$ largest relative error of $y_{1}, y_{2}$ and $y_{3} n$ at $x=2$ and $x=48$ as a function of the stepsize $h$
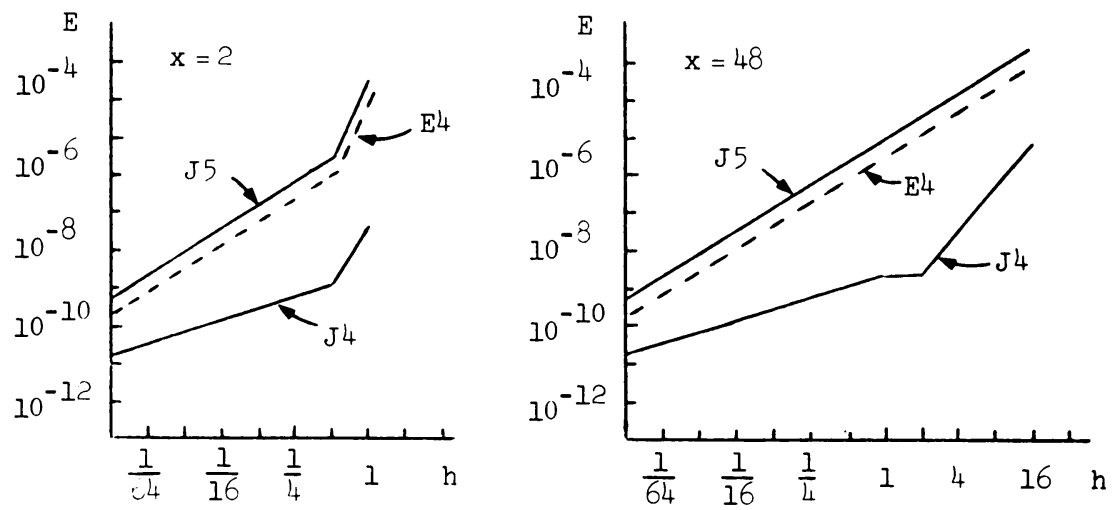

for E4. However, for $h>2$ the order of damping at infinity seems to come into play. This change of behavior occurs in this example only for relatively large $h$; however, in the following example it occurs for some $h$ smaller than $2^{-7}$.

Example 6. The following stiff initial value problem arose from a chemistry problem (see Gear [18]).

$$
\begin{aligned}
& y_{1}^{\prime}(x)=-0.013 y_{2}(x)-1000 y_{1}(x) y_{2}(x)-2500 y_{1}(x) y_{3}(x), \\
& y_{2}^{\prime}(x)=-0.013 y_{2}(x)-1000 y_{1}(x) y_{2}(x), \\
& y_{3}^{\prime}(x)=-2500 y_{1}(x) y_{3}(x),
\end{aligned}
$$

with $y_{1}(0)=0, y_{2}(0)=1, y_{3}(0)=1$. For $x=2$ and $x=48$ the exact solution is

$$
\begin{array}{ll}
y_{1}(2)=-0.3616933169289(-5), & y_{1}(48)=-.1945338956808(-5), \\
y_{2}(2)=0.9815029948230, & y_{2}(48)=0.6110474831446 \\
y_{3}(2)=1.018493388244, & y_{3}(48)=1.388950571516 .
\end{array}
$$

These values are correct to 1.5 units of the last given digit. The eigenvalues of the Jacobian (along the exact solution) are

$$
\lambda_{1}(x) \equiv 0, \lambda_{2}(x) \in[-0.01,0], \lambda_{3}(x) \in[-4200,-3499] \text { for } x \in[0,48] .
$$

A constant step $h$ is used throughout the integration. The methods are started using the SSP-routine DRKGS with an error tolerance $10^{-14}$. The nonlinear equation (2-1) is solved using Newton's method. As first approximation to $y_{i n+2}$ the value $y_{i n+1}^{2} / y_{i n}$ has been used, where $y_{i n}$ corresponds to $y_{i}\left(x_{n}\right)$. The Newton iteration was terminated as soon as the relative change in each component was less than $10^{-12}$. The number of Newton steps was always the same for all three methods. In Table 6 the total number of Newton iterations and the average number of Newton iterations used in one itegration step is given.

In Figure 5 the largest of the relative errors in the three components are given as a function of $h=2^{n}$ at $x=2$ and $x=48$. One should appreciate the fact that the relative error was always in all components approximately the same despite the fact that $y_{1}(x) 10^{5} \sim y_{2}(x), x \in[1,48]$. For example, we found with $\mathrm{J} 4, h=2$ 
at $x=48$ the relative errors $2.3 \times 10^{-9}, 1.9 \times 10^{-9}$ and $0.8 \times 10^{-9}$ for the first, second and third component, respectively. Observe that the two methods with $\epsilon=1 / 2$ behave in the same way, despite the fact that one is of error order 4 and the other of error order 5. Moreover, J4 with $\epsilon=1$ behaves differently from E4 with $\epsilon=1 / 2$; but both have error order 4 . For example, to get a relative error of at most $10^{-8}$ at $x=$ 48 the number of Newton iterations used by E4 is 20 times larger than the one used by J4. The calculations have been performed on an IBM 360 using double precision (i.e. 56-bit mantissa).

Acknowledgement. The author would like to thank Dalhousie University for providing him with a Killam Postdoctoral Fellowship. A large part of this work was carried out when the author stayed at Dalhousie University. We would like to thank the referee for his useful remarks which improved the exposition of the article considerably.

Institute for Mathematics Ruhr-University Bochum 4630 Bochum, West Germany

1. L. V. AHLFORS, Complex Analysis, McGraw-Hill, New York, 1953. MR 14, 857 .

2. O. AXELSSON, “A class of $A$-stable methods," $B I T$, v. 9, 1969, pp. 185-199. MR 40 \#8266.

3. G. BIRKHOFF \& R. S. VARGA, "Discretization errors for well-set Cauchy problems. I," J. Math. and Phys., v. 44, 1965, pp. 1-23. MR 31 \#189.

4. J. L. BLUE \& H. K. GUMMEL, "Rational approximations to matrix exponential for systems of stiff differential equations," J. Computational Phys., v. 5, 1970, pp. 70-83. MR 40 \#8267.

5. D. A. CALAHAN, "Numerical solution of linear systems with widely separated time constants," Proc. IEEE, Nov., 1967,.pp. 2016-2017.

6. J. C. CAVENDISH, W. E. CULHAM \& R. S. VARGA, "A comparison of Crank-

Nicolson and Chebyshev rational methods for numerically solving linear parabolic equations," $J$. Computational Phys., v. 10, 1972, pp. 354-368. MR 48 \#3268.

7. F. H. CHIPMAN, Numerical Solution of Initial Value Problems Using A-Stable RungeKutta Processes, Research Report CSRR 2042, Dept. of A.A.C.S., Univ. of Waterloo, 1971.

8. C. W. CRYER, "On the instability of high order backward-difference multistep methods," $B I T$, v. 12, 1972, pp. 17-25. MR $46 \# 10208$.

9. G. G. DAHLQUIST, "Convergence and stability in the numerical integration of ordinary differential equations," Math. Scand., v. 4, 1956, pp. 33-53. MR 18, 338.

10. G. G. DAHLQUIST, "Stability and error bounds in the numerical integration of ordinary differential equations," Kungl. Tekn. Högsk. Handl. Stockholm, No. 130, 1959. MR 21 \#1706.

11. G. G. DAHLQUIST, “A special stability problem for linear multistep methods," $B I T$, v. 3, 1963, pp. 27-43. MR $30 \# 715$.

12. J. W. DANIEL \& R. E. MOORE, Computation and Theory in Ordinary Differential Equations, Freeman, San Francisco, Calif., 1970. MR 42 \#2667.

13. E. J. DAVISON, "A high-order Crank-Nicholson technique for solving differential equations," Comput. J., v. 10, 1967, pp. 195-197. MR 35\#5141.

14. B. L. EHLE, On Padé Approximations to the Exponential Function and A-Stable Methods for the Numerical Solution of Initial Value Problems, Research Report CSRR 2010, Dept. of A.A.C.S., Univ. of Waterloo, 1969.

15. M. E. ENGELI, Symbal, Summary and Examples, Fides, Union Fiduciaire, Zürich, 1970.

16. W. ENRIGHT, Studies in the Numerical Solution of Stiff Ordinary Differential

Equations, Technical Report No. 46, Dept. of C. S., Univ. of Toronto, 1972.

17. T. FORT, Finite Differences and Difference Equations in the Real Domain, Clarendon Press, Oxford, 1948. MR 9, 514. 
18. C. W. GEAR, "The automatic integration of stiff ordinary differential equations," Information Processing 68 (Proc. IFIP Congress, Edinburgh, 1968), Vol I: Mathematics, Software, edited by A. J. H. Morell, North-Holland, Amsterdam, 1969, pp. 187-193. MR 41 \#4808. 19. C. W. GEAR, Numerical Initial Value Problems in Ordinary Differential Equations, Prentice-Hall, Englewood Cliffs, N.J., 1971. MR 47 \#447.

20. E. GRIEPENTROG, "Mehrschrittverfahren zur numerischen Integration von gewöhnlichen Differentialgleichungssystemen und asymptotische Exaktheit," Wiss. Z. HumboltUniv. Berlin Math.-Natur. Reine, v. 19, 1970, pp. 637-653. MR 47 \#9833.

21. P. HENRICI, Discrete Variable Methods in Ordinary Differential Equations, Wiley, New York, 1962. MR 24 \#B1772.

22. K. HENSEL \& G. LANDSBERG, Theorie der algebraischen Funktionen einer Variablen, Teubner, Leipzig, 1902.

23. R. JELTSCH, "Integration of iterated integrals by multistep methods," Numer. Math., v. 21, 1973/74, pp. 303-316. MR $49 \# 1786$.

24. R. JELTSCH, Multistep Multiderivative Methods and Hermite-Birkhoff Interpolation, Proc. Fifth Manitoba Conf. on Numerical Mathematics (Univ. of Manitoba, Winnipeg, Man., 1975), pp. 417-428. Congressus Numerantium, No. XVI, Utilitas Math. Publ., Winnipeg, Man., 1976.

25. J. D. LAMBERT, Computational Methods in Ordinary Differential Equations, Wiley, London, 1973.

26. J. D. LAMBERT \& A. R. MITCHELL, "On the solution of $y^{\prime}=f(x, y)$ by a class of high accuracy difference formulae of low order," Z. Angew. Math. Phys., v. 13, 1962, pp. 223-232 MR 25 \#3610.

27. J. D. LAWSON, Order Constrained Best Rational Approximation to $\exp (x)$ on $(-\infty, 0]$. (Private communication.)

28. W. LINIGER \& R. A. WILLOUGHBY, Efficient Integration Methods for Stiff Systems of Ordinary Differential Equations, IBM Research Report RC 1970, 1967.

29. F. R. LOSCALZO, On the Use of Spline Functions for the Numerical Solution of Ordinary Differential Equations, MRC Technical Summary Report No. 869, Univ. of Wisconsin, May 1968.

30. G. J. MAKINSON, "Stable high order implicit methods for the numerical solution of systems of differential equations," Comput. J., v. 11, 1968/69, pp. 305-310. MR 38 \#4040.

31. M. MARDEN, Geometry of Polynomials, 2nd ed., Math. Surveys, no. 3, Amer. Math. Soc., Providence, R.I., 1966. MR 37 \#1562.

32. R. E. MOORE, "The automatic analysis and control of error in digital computation based on the use of interval numbers," in Error in Digital Computation, Vol. 1 (Proc. Advanced Sem., Madison, Wis., 1964), edited by L. Rall, Wiley, New York, 1965, pp. 61-130.

MR 31 \#886.

33. M. R. OSBORNE, "A new method for the integration of stiff systems of ordinary differential equations," Information Processing 68 (Proc. IFIP Congress, Edinburgh, 1968), Vol. I: Mathematics, Software, edited by A. J. H. Morell, North-Holland, Amsterdam, 1969, pp. $200-204$. MR $41 \# 4810$.

34. A. PFLUGER, Theorie der Riemannschen Flächen, Springer-Verlag, Berlin, 1957. MR 18, 796.

35. M. REIMER, “Finite difference forms containing derivatives of higher order," SIAM J. Numer. Anal., v. 5, 1968, pp. 725-738. MR 40 \#3742.

36. H. J. STETTER, Analysis of Discretization Methods of Ordinary Differential Equations, Springer-Verlag, New York, 1973.

37. W. E. THOMPSON, "Solution of linear differential equations," Comput. J., v. 10, 1968 , pp. 417-418.

38. R. S. VARGA, "Some results in approximation theory with applications to numerical analysis," in Numerical Solution of Partial Differential Equations, II (SYNSPADE 1970), (Proc. Sympos., Univ. of Maryland, 1970), edited by B. E. Hubbard, Academic Press, New York, 1971, pp. 623-649.

39. O. B. WIDLUND, "A note on unconditionally stable linear multistep methods," BIT, v. 7, 1967, pp. 65-70. MR 35 \#6373. 